\title{
Mit der Sprache ging es immer schon bergab \\ Dynamik, Wandel und Variation aus sprachhistorischer Perspektive
}

\begin{abstract}
Die Vorstellung eines Verfalls der deutschen Sprache lässt sich mindestens bis in das 16. Jahrhundert zurückverfolgen, als Schulmeister sich beschwert haben, dass ihre Schüler wegen der um sich greifenden Variation nicht mehr wüssten, was korrektes Deutsch sei. Ähnliche Vorstellungen treten etwa gleichzeitig in anderen europäischen Ländern auf und können vielleicht mit dem langsamen Ersatz des Lateins als vorherrschender Sprache des Schrifttums und der Bildung in Zusammenhang gebracht werden. Sie beruhen auf verbreiteten irrtümlichen Annahmen über das Wesen der Sprache, insbesondere dass die zugrundeliegende Form jeder Sprache homogen und unwandelbar sei und seit sehr langem - eventuell seit Babel - so existiert habe. Diese Annahmen muss man mit Watts (2011) als Mythen werten, sie sind jedoch sehr beharrlich, und in der frühen Neuzeit dienten sie als Grundlage für die Erschaffung der heutigen deutschen Standardsprache, die aus diesem Grunde genauso wie alle anderen europäischen Kultur- oder Standardsprachen eigentlich als ein rezentes kulturelles Artefakt anzusehen ist.

In diesem Beitrag wird anhand von Material aus einem neuen elektronischen Korpus der deutschen Sprache des 17. und 18. Jahrhunderts gezeigt, wie die Standardsprache entstanden ist als Ergebnis dieser Annahmen sowie aus der Vorstellung, nur auf diese Weise sei die deutsche Sprache vor dem endgültigen Verfall zu retten. Im Laufe dieses Vorgangs wurde wo möglich jede Variation aus der Schriftsprache eliminiert und dabei auch sprachliche Varianten stigmatisiert, die heute noch häufig sind, auch wenn sie als „,substandard“, „,nicht korrekt“ oder „nicht hochsprachlich“ gelten. Auch wurden Regeln des „guten“ hochdeutschen Sprachgebrauchs festgelegt (oder erdacht), die Muttersprachler im spontanen Gespräch immer noch kaum beachten. Aber die Sprachgeschichte lehrt, dass Variation und Wandel nicht zum Verfall der Sprache führen, sondern die dynamische Flexibilität gewährleisten, die für die Sprache nötig ist, wenn sie allen sozial und kulturell erforderlichen Bedürfnissen der menschlichen Kommunikation gerecht werden muss.
\end{abstract}

\section{Einleitung: Das GerManC-Korpus}

Von 2006 bis 2012 wurde an der Universität Manchester ein elektronisches Korpus des frühmodernen Deutsch (d.h. zwischen den Jahren 1650 und 1800) von etwa einer Million Wortformen erstellt. ${ }^{1}$ Diese Periode ist bisher

Das Gesamtkorpus, mit genauen Angaben über die aufgenommenen Texte, ist im Oxford Text Archive gespeichert und darf ohne Einschränkung heruntergeladen werden: www.ota.ox. ac.uk/desc/2544. Weitere Information zum Korpus findet man bei Bennett et al. (2011) sowie auf der Projektwebseite: www.alc.manchester.ac.uk/subjects/german/research/projects/ germanc/. 
trotz ihrer Bedeutung für die endgültige Standardisierung der deutschen Sprache sprachhistorisch relativ wenig erforscht worden, und ein wichtiges Ziel des Korpus war es, diesen Mangel beheben zu helfen, indem es der Forschung zum ersten Mal eine leicht zugängliche Sammlung von Sprachmaterial zur Verfügung stellen sollte, die für den überlieferten Sprachgebrauch dieser Epoche möglichst repräsentativ wäre. Entsprechend dieser Zielsetzung besteht das Korpus aus etwa 360 Textauszügen von jeweils 2000 Wörtern aus den acht Textsorten, die in dieser Periode ausreichend belegt sind, mit einer gleichen Anzahl von Texten aus fünf sprachlichen Großräumen und drei Unterperioden von jeweils 50 Jahren, in die die ganze Periode von anderthalb Jahrhunderten aufgeteilt wurde.

Dieses Korpus stellt eine wichtige neue Ressource zur Erforschung der Entwicklung der deutschen Sprache in der frühen Neuzeit dar, vor allem wegen der Berücksichtigung von bisher etwas weniger beachteten Textsorten. Z.B. hat Polenz (2000, S. 18) schon darauf hingewiesen, dass

Zeitungen [...] - nach der Luther-Bibel - auch zum wirksamsten Mittel der Popularisierung und Verbreitung einheitlicher Sprachvarianten auf dem Wege zur nationalen Schriftsprache [wurden],

aber systematische Untersuchungen von deren sprachlichen Eigenarten sind immer noch etwas spärlich. Sie bieten jedoch viel aufschlussreiches Sprachmaterial. Man liest z.B. in einer Münchner Zeitung (Mercurij-Relation: Extract-Schreiben auß Lintz [...]) vom Juli 1684:

Von der jüngst bey Waitzen erhaltenen Victori berichte über voriges/ daß die Türcken über 25000. Mann starck gewesen/ nit allein Jhro Durchl. dem Hertzogen von Lothringen sein Roß/ sondern auch 2. Cavallieren neben seiner die Pferdt todt geschossen worden.

Man könnte natürlich annehmen, der Schreiber habe mit dem Hertzogen von Lothringen sein $\mathrm{Ro} \beta$ möglicherweise einen vom Standpunkt der grammatischen Korrektheit keineswegs zu beanstandenden dativus incommodi gesetzt, aber da es nicht leicht ist, die verschiedenen Verwendungsmöglichkeiten des Dativ auseinanderzuhalten, vgl. Zifonun/Hoffmann/Strecker (1997, S. 1337-1341), ist die Analyse nicht gänzlich von der Hand zu weisen, dass es sich hier um eben denjenigen schlampigen Gebrauch des Dativs handelt, der nach Sick (2004) den Tod des Genitivs bedeuten könnte. Nun ist der Genitiv, anders als das Ross des Herzogs, noch lange nicht tot. Es besteht aber immer noch eine Variation, die auch schon im 17. Jahrhundert existiert hat, und zwar kann man den Besitz durch den Genitiv oder durch eine Dativparaphrase ausdrücken. Die letztere Variante wird jetzt aber als „nicht korrekt" oder „nicht hochsprachlich“ angesehen, und sie gilt insbesondere im geschriebenen Deutsch als absolut inakzeptabel, obwohl sie von vielen Muttersprachlern - vielleicht sogar von einer großen Mehrheit - täglich verwendet wird. 


\section{Zur Entstehung der deutschen Standardsprache und zu verbreiteten Mythen über die Sprache}

Wenn jedoch eine sprachliche Erscheinung wie die Dativparaphrase seit über dreihundert Jahren existiert - mindestens, denn Belege dafür lassen sich möglicherweise bis in ahd. Zeit zurückverfolgen, vgl. Fleischer (2011, S. 96-99) - ohne dass die konkurrierende Variante mit dem Genitiv endgültig dadurch ersetzt wurde, kann man sie kaum als glaubhaftes Beispiel für den heutigen Verfall der Sprache werten. Wir haben es hier eher mit einer Änderung in der Einstellung zur deutschen Sprache zu tun, die wir zunächst durch eine etwa 150 Jahre frühere Aussage erläutern können. Kluge (1904, S. 56) führt nämlich die folgende Aussage eines Erfurter Schulmeisters, Meister Hans Fabritius, aus dem Jahre 1531 auf:

Ich weiß schier nicht, wie ich meine Schulers lehren soll der Ursachen halben, daß jetzunder, wo unser nur drei oder vier deutsche Schreibers zusamen koment, hat jeder einen sonderlichen Gebrauch. Wolte Gott, das es darhin komen möchte, daß die Kunst des Schreibens einmal wider in ein rechten Prauch komen möcht.

Mit nur relativ leichten Änderungen könnte man sich dies als die Aussage eines heutigen Deutschlehrers vorstellen, der über den Verfall der Sprache und deren Verwahrlosung durch die Jugend klagt. Es lohnt sich jedoch, auf diese Aussage etwas gründlicher einzugehen. Im Jahre 1531 gab es nämlich keine einheitliche deutsche Sprache, vgl. Durrell (1999), und es hatte nie eine solche gegeben, aber anscheinend hat Hans Fabritius es als absolut selbstverständlich angenommen, dass sie seit langem existierte und es ihm als Lehrer oblag, seinen „Schulers“ die Normen dieser Einheitssprache beizubringen. Diese Vorstellung, dass jede Sprache eine unveränderliche Grundform besitzt, hat in der Entwicklung der modernen europäischen Standardsprachen eine grundsätzliche Rolle gespielt. Sie tritt in allen europäischen Sprachgebieten etwa gleichzeitig auf, und zwar am Anfang der frühen Neuzeit, als die Volkssprachen nach der Verbreitung des Buchdrucks und der Reformation anfingen, allmählich an der Alleinherrschaft des Lateins als Kultursprache zu rütteln und, wie von Hans Fabritius, auch zum ersten Mal systematisch unterrichtet wurden. Watts $(2011,2012)$ bezeichnet diese Vorstellung als den „Mythos der sprachlichen Homogenität“, von dem auch eine Anzahl weiterer sprachlicher Mythen abhängt, insbesondere der Mythos einer althergebrachten, „reinen“ Sprache. Diese Mythen ${ }^{2}$ scheinen der Aussage von Fabritius zugrundezuliegen, indem er unreflektiert voraussetzt, dass

In der Diskussion nach dem Vortrag wurde darauf hingewiesen, dass das deutsche Wort Mythos nicht unbedingt das gleiche aussage, wie Englisch myth. Jedoch scheint die bei Duden (2001, S. 1113) angeführte Definition von Mythos auch den hier intendierten Sinn einzuschließen, und da Maitz (2010) Mythos auch in einer durchaus vergleichbaren Bedeutung in einem sprachwissenschaftlichen Kontext verwendet, halte ich den Gebrauch dieses Terminus hier weiterhin für gerechtfertigt. 
die deutsche Sprache eine homogene Grundform hat, die allein gültig ist, die in dieser festgelegten und unabänderlichen Form seit Urzeiten existiert und die durch die sündhaft nachlässige Menschheit (bzw. die Jugend) in Verfall geraten ist. Wir werden sehen, dass diese Mythen und der ihnen zugrundeliegende ahistorische Sprachbegriff heute noch weit verbreitete landläufige Auffassungen über die Sprache bestimmen und, wie Maitz (2010) und andere schon gezeigt haben, immer noch Anlass geben zu Äußerungen, die mit den Beschwerden von Hans Fabritius durchaus vergleichbar sind. Milroy/Milroy (1999, S. 24-46) sprechen in diesem Zusammenhang von einer „Complaint Tradition“, die sie bis William Caxton, der den Buchdruck in England eingeführt hat, zurückverfolgen. Diese Mythen haben, wie Watts (2011) zeigt, bei der Entstehung der englischen Standardsprache ausschlaggebend gewirkt, und sie waren auch in der Geschichte der deutschen Sprache kaum minder bedeutend. Im Grunde genommen sind alle europäischen Standardsprachen aus dem Versuch im 17. und 18. Jahrhundert entstanden, die ursprüngliche, homogene, echte Sprache aufzufinden und sie möglicherweise so festzulegen, dass sie vor dem weiteren Verfall bewahrt wird. Auf diese Weise sind sie letztlich, wie Haugen (1966) erkannt hat, keine natürlich entstandenen Sprachformen, sondern „kulturelle Artefakte“, die schreibsprachlichen Konstrukte einer Bildungselite.

Dies wurde in Deutschland lange vehement geleugnet, vor allem aus ideologischen Gründen, denn im Zeitalter des aufkommenden Nationalismus wollte man nicht wahrhaben, dass die deutsche Muttersprache, die für die Nationbildung einen nicht zu unterschätzenden Symbolwert hatte, nicht grundsätzlich aus der Sprache des Volks hervorgekommen war. So schrieb Gutjahr (1910, S. 6):

Alle bisherigen Versuche, die Lösung des Problems der Entstehung der neuhochdeutschen Schriftsprache zumeist im Rahmen eines künstlichen Geschaffenseins auf dem Papier herbeizuführen, können in keinerlei Weise befriedigen.

Dieser Gedanke wurde dann bekanntlich von Theodor Frings mit seiner These eines Ursprungs der neuhochdeutschen Schriftsprache in den Siedlermundarten der im Mittelalter kolonisierten deutschen Ostgebiete weitergeführt, aber diese wurde von Werner Besch und anderen Forschern von den 1960er Jahren an endgültig widerlegt, indem sie gezeigt haben, dass der Standardisierungsprozess tatsächlich vornehmlich im Schriftlichen vor sich ging, vgl. Durrell (1999, 2001). Keller (1978, S. 371) formuliert den Vorgang treffend in direktem Widerspruch zu Gutjahr: „what was happening was happening primarily on paper"“.

Im Laufe des 17. und 18. Jahrhunderts wurde dieser in frühneuhochdeutscher Zeit begonnene Prozess weitergeführt, indem nach dem bekannten Standardisierungsmodell von Haugen (1966) bestimmte sprachliche Varianten als ,korrekt" selegiert und kodifiziert wurden, d.h. sie konnten mit 
Vorstellungen über die „richtige“, „ursprüngliche“ deutsche Sprache vereinbart werden. Träger dieses Prozesses war vor allem eine soziale Elite von Literaten und Sprachexperten (Polenz 2000, S. 135), die ihrem Wirken weitgehend die von Watts (2011) identifizierten Mythen zugrundelegten. Auf dieser Basis wurde eine Anzahl von charakteristischerweise relativ vagen und uneinheitlichen Kriterien und Voraussetzungen entwickelt, nach denen sprachliche Formen bewertet wurden. Insbesondere galt Variation an sich als verwerflich, denn sie gingen von der grundlegenden Annahme sprachlicher Homogenität aus, und so musste eine von zwei konkurrierenden Formen selbstverständlich „,falsch“ oder ,minderwertig“" sein.

\section{Korpusbeispiele für die Eliminierung sprachlicher Variation in der frühen Neuzeit und für die Stigmatisierung bestimmter Varianten}

Dieser Prozess lässt sich sehr gut durch Material aus dem GerManC-Korpus von Texten aus dem 17. und 18. Jahrhundert veranschaulichen; dazu sollen nun ein paar geeignete Beispiele herausgegriffen werden. Mitte des 17. Jahrhunderts war sprachliche Variation noch eher die Regel als die Ausnahme, vor allem wenn man ein repräsentatives Sample von Textsorten und Regionen in Betracht zieht, und sie betraf nicht nur die Orthographie, sondern auch wesentliche Gebiete der Grammatik wie die Flexion.

\subsection{Die Flexion des schwachen Adjektivs im Nominativ/Akkusativ Plural}

In dieser Zeit, wie schon im Frühneuhochdeutschen, kommt beim „schwachen" Adjektiv im Nominativ/Akkusativ Plural neben der heute verbindlichen Endung -en auch - $e$ vor, vgl. Ebert et al. (1993, S. 189-199) und Solms/Wegera (1991, S. 175-184). Diese Variation ist nicht alt, denn das Mittelhochdeutsche kannte nur das ererbte Flexiv -en, aber vor allem im oberdeutschen Raum erscheint dann etwa seit Mitte des 16. Jahrhunderts die Variante $-e$, und diese wird bis Ende der frühneuhochdeutschen Periode im ganzen hochdeutschen Raum vorherrschend mit Ausnahme des Ostmitteldeutschen. Solms/Wegera (ebd., S. 176) werten die Durchsetzung von -en im 18. Jahrhundert und die Bestimmung dieser Variante als alleingültiger hochsprachlicher Norm als die Verallgemeinerung eines in erster Linie ostmitteldeutschen Usus, wobei literarische Sprachvorbilder maßgeblich gewirkt hätten. Das in Tabelle 1 angegebene Material unseres Korpus, das die Endungen des schwachen Adjektivs nach einem bestimmten Artikel angibt, scheint diese Annahme zu unterstützen, denn die Variante -en, die 
in der zweiten Hälfte des 17. Jahrhunderts nur im omd. Raum mit bedeutender Mehrheit vorherrschend war, breitete sich im Laufe der nächsten hundert Jahre auf das gesamte Sprachgebiet aus. ${ }^{3}$

\begin{tabular}{|c|c|c|c|c|c|c|}
\hline & \multicolumn{2}{|c|}{$1650-1700$} & \multicolumn{2}{|c|}{ 1700-1750 } & \multicolumn{2}{|c|}{$1750-1800$} \\
\hline & $-e$ & -en & $-e$ & $-e n$ & $-e$ & -en \\
\hline Norddt. & $\begin{array}{c}72 \\
(42 \%)\end{array}$ & $\begin{array}{c}101 \\
(58 \%)\end{array}$ & $\begin{array}{c}45 \\
(26 \%)\end{array}$ & $\begin{array}{c}126 \\
(74 \%)\end{array}$ & $\begin{array}{c}24 \\
(15 \%)\end{array}$ & $\begin{array}{c}138 \\
(85 \%)\end{array}$ \\
\hline Westmitteldt. & $\begin{array}{c}85 \\
(51 \%)\end{array}$ & $\begin{array}{c}81 \\
(49 \%)\end{array}$ & $\begin{array}{c}77 \\
(47 \%)\end{array}$ & $\begin{array}{c}86 \\
(53 \%)\end{array}$ & $\begin{array}{c}35 \\
(24 \%)\end{array}$ & $\begin{array}{c}108 \\
(76 \%)\end{array}$ \\
\hline Ostmitteldt. & $\begin{array}{c}28 \\
(21 \%)\end{array}$ & $\begin{array}{c}108 \\
(79 \%)\end{array}$ & $\begin{array}{c}48 \\
(29 \%)\end{array}$ & $\begin{array}{c}116 \\
(71 \%)\end{array}$ & $\begin{array}{c}16 \\
(9 \%)\end{array}$ & $\begin{array}{c}163 \\
(91 \%)\end{array}$ \\
\hline Westoberdt. & $\begin{array}{c}84 \\
(50 \%)\end{array}$ & $\begin{array}{c}83 \\
(50 \%)\end{array}$ & $\begin{array}{c}89 \\
(52 \%)\end{array}$ & $\begin{array}{c}81 \\
(48 \%)\end{array}$ & $\begin{array}{c}55 \\
(29 \%)\end{array}$ & $\begin{array}{c}133 \\
(71 \%)\end{array}$ \\
\hline Ostoberdt. & $\begin{array}{c}102 \\
(60 \%)\end{array}$ & $\begin{array}{c}68 \\
(40 \%)\end{array}$ & $\begin{array}{c}107 \\
(64 \%)\end{array}$ & $\begin{array}{c}61 \\
(36 \%)\end{array}$ & $\begin{array}{c}46 \\
(28 \%)\end{array}$ & $\begin{array}{c}119 \\
(72 \%)\end{array}$ \\
\hline
\end{tabular}

Tab. 1: Die schwache Adjektivflexion im Nom./Akk. Plural (Adjektiv nach bestimmtem Artikel), Korpusbelege nach Regionen

Wenn wir jedoch die Belege nach Textsorten sortieren, scheint das die von Voeste (1999b) geäußerten Zweifel über die absolute Vorbildwirkung von literarischen Texten und dem ostmitteldeutschen Raum zu bekräftigen. In Tabelle 2 sehen wir, dass das Verhältnis der Variante -en zu - $e$ in der ersten Hälfte des 18. Jahrhunderts in den literarischen Gattungen im Vergleich zum späten 17. Jahrhundert leicht zurückgeht, wohingegen das Übergewicht von -en in den geisteswissenschaftlichen Texten und den Briefen auffällt, so dass der Schluss nahe liegen dürfte, dass es vor allem das neue Bildungsbürgertum war, das sich für die Variante -en entschied und sie am frühesten allgemein adoptierte. Aber deutlich hielt die Variation viel länger an, als man bisher vermutet hat, so dass noch in der zweiten Hälfte des 18. Jahrhunderts die „richtige“ Variante noch nicht alleinherrschend war. Juristische Texte erscheinen in dieser Hinsicht ausgesprochen konservativ in ihrem Sprachgebrauch, aber, wie die Korpusbelege bestätigen, kommen beide Flexive z.B. noch in Goethes „Egmont“" vor. Wie Voeste (1999a) klarmacht,

Frühere Darstellungen zu diesem Thema auf der Basis von Material aus dem GerManCKorpus finden sich bei Durrell/Ennslin/Bennett (2008) und Bennett et al. (2011). Die quantitativen Angaben in diesen Arbeiten unterscheiden sich geringfügig von denen in diesem Beitrag, weil sie auf vorläufigen Ergebnissen von früheren, nicht ganz vollständigen Versionen des Korpus beruhen. 
waren diese Normierungsprozesse oft hochkomplex, und die Gründe für die endgültige Selegierung der einen oder der anderen Variante sind selten mit absoluter Sicherheit nachweisbar oder sogar nachvollziehbar. Es genügte keineswegs, dass eine Variante im Ostmitteldeutschen oder in literarischen Texten vorherrschend war, aber wenn tatsächlich eine frühe Adoption der Variante -en durch das Bildungsbürgertum erfolgt ist, könnte man in diesem Fall auch das Einwirken einflussreicher Grammatiker und Sprachtheoretiker in Betracht ziehen, die mehrheitlich -en favorisierten, vgl. Voeste (1999a). Jedoch sind die Kriterien, warum einer bestimmten Variante der Vorzug gegeben wurde, recht vage, subjektiv oder arbiträr, und anscheinend genügte es einfach, dass sie aus irgendeinem Grund nicht gefiel - oder sie stimmte vielleicht nicht mit dem eigenen Gebrauch überein - um sie für „falsch“ zu erklären, und in dieser Hinsicht sind diese Grammatiker durchaus mit den Sprachwächtern der Gegenwart zu vergleichen.

\begin{tabular}{|c|c|c|c|c|c|c|}
\hline & \multicolumn{2}{|c|}{$1650-1700$} & \multicolumn{2}{|c|}{$1700-1750$} & \multicolumn{2}{|c|}{$1750-1800$} \\
\hline & $-e$ & $-e n$ & $-e$ & -en & $-e$ & -en \\
\hline Zeitungen & $\begin{array}{c}135 \\
(74 \%)\end{array}$ & $\begin{array}{c}48 \\
(26 \%)\end{array}$ & $\begin{array}{c}71 \\
(48 \%)\end{array}$ & $\begin{array}{c}78 \\
(52 \%)\end{array}$ & $\begin{array}{c}25 \\
(15 \%)\end{array}$ & $\begin{array}{c}141 \\
(85 \%)\end{array}$ \\
\hline $\begin{array}{l}\text { Juristische } \\
\text { Texte }\end{array}$ & $\begin{array}{c}43 \\
(52 \%)\end{array}$ & $\begin{array}{c}40 \\
(48 \%)\end{array}$ & $\begin{array}{c}81 \\
(74 \%)\end{array}$ & $\begin{array}{c}29 \\
(26 \%)\end{array}$ & $\begin{array}{c}66 \\
(54 \%)\end{array}$ & $\begin{array}{c}56 \\
(46 \%)\end{array}$ \\
\hline $\begin{array}{l}\text { Geisteswiss. } \\
\text { Texte }\end{array}$ & $\begin{array}{c}48 \\
(48 \%)\end{array}$ & $\begin{array}{c}52 \\
(52 \%)\end{array}$ & $\begin{array}{c}25 \\
(25 \%)\end{array}$ & $\begin{array}{c}75 \\
(75 \%)\end{array}$ & $\begin{array}{c}13 \\
(15 \%)\end{array}$ & $\begin{array}{c}75 \\
(85 \%)\end{array}$ \\
\hline Briefe & $\begin{array}{c}25 \\
(48 \%)\end{array}$ & $\begin{array}{c}27 \\
(52 \%)\end{array}$ & $\begin{array}{c}26 \\
(29 \%)\end{array}$ & $\begin{array}{c}65 \\
(71 \%)\end{array}$ & $\begin{array}{c}14 \\
(20 \%)\end{array}$ & $\begin{array}{c}55 \\
\left(80^{\circ} \%\right)\end{array}$ \\
\hline Predigten & $\begin{array}{c}39 \\
(38 \%)\end{array}$ & $\begin{array}{c}65 \\
(62 \%)\end{array}$ & $\begin{array}{c}43 \\
(41 \%)\end{array}$ & $\begin{array}{c}62 \\
(59 \%)\end{array}$ & $\begin{array}{c}26 \\
(35 \%)\end{array}$ & $\begin{array}{c}49 \\
(65 \%)\end{array}$ \\
\hline $\begin{array}{l}\text { Naturwiss. } \\
\text { Texte }\end{array}$ & $\begin{array}{c}47 \\
(33 \%)\end{array}$ & $\begin{array}{c}95 \\
(67 \%)\end{array}$ & $\begin{array}{c}59 \\
(47 \%)\end{array}$ & $\begin{array}{c}66 \\
(53 \%)\end{array}$ & $\begin{array}{c}18 \\
(11 \%)\end{array}$ & $\begin{array}{c}143 \\
(89 \%)\end{array}$ \\
\hline $\begin{array}{l}\text { Erzählende } \\
\text { Prosa }\end{array}$ & $\begin{array}{c}29 \\
(33 \%)\end{array}$ & $\begin{array}{c}58 \\
(67 \%)\end{array}$ & $\begin{array}{c}32 \\
(40 \%)\end{array}$ & $\begin{array}{c}48 \\
(60 \%)\end{array}$ & $\begin{array}{c}9 \\
(11 \%)\end{array}$ & $\begin{array}{c}71 \\
(89 \%)\end{array}$ \\
\hline Dramen & $\begin{array}{c}15 \\
(23 \%)\end{array}$ & $\begin{array}{c}50 \\
(77 \%)\end{array}$ & $\begin{array}{c}28 \\
(36 \%)\end{array}$ & $\begin{array}{c}49 \\
(64 \%)\end{array}$ & $\begin{array}{c}6 \\
(13 \%)\end{array}$ & $\begin{array}{c}42 \\
(87 \%)\end{array}$ \\
\hline Gesamt & $\begin{array}{c}381 \\
(47 \%)\end{array}$ & $\begin{array}{c}435 \\
(53 \%)\end{array}$ & $\begin{array}{c}365 \\
(44 \%)\end{array}$ & $\begin{array}{c}472 \\
(56 \%)\end{array}$ & $\begin{array}{c}177 \\
(22 \%)\end{array}$ & $\begin{array}{c}632 \\
(78 \%)\end{array}$ \\
\hline
\end{tabular}

Tab. 2: Die schwache Adjektivflexion im Nom./Akk. Plural (Adjektiv nach bestimmtem Artikel), Korpusbelege nach Textsorten 


\subsection{Die Genusdifferenzierung bei dem Zahlwort zwei}

Beim nächsten Fall, der hier besprochen werden soll, und zwar dem Abbau der Genusdifferenzierung beim Zahlwort zwei, war dagegen ein Präskriptionsversuch von Grammatikern und Sprachtheoretikern letztlich nicht erfolgreich. Bis in die frühe Neuzeit hatte das Zahlwort zwei genusdifferenzierte Formen im Nominativ und Akkusativ, vgl. Walch/Häckel (1988, S. 536-552). Wie Tabelle 3 zeigt, ist es in unserem Korpus leider spärlich belegt, aber einige vorläufige Schlüsse dürfen wohl aus diesem Material gezogen werden. Es scheint erstens klar zu sein, dass der Ausgleich im späten 17. Jahrhundert eigentlich schon weiter vorangeschritten war als bisher angenommen. Belege für eine „ungeregelte Vermischung“ der Formen, vgl. Schirmunski (1962, S. 474), sind dann schon in allen Regionen anzutreffen, und in kaum einem einzigen Text in unserem Korpus ist eine absolut konsequente Handhabung der Genusdifferenzierung vorzufinden. Dagegen haben unsere ostmitteldeutschen Texte nach 1700 fast ausschließlich genusindifferentes zwei.

\begin{tabular}{|c|c|c|c|c|c|c|c|c|c|c|c|c|c|c|c|}
\hline \multirow[b]{3}{*}{$\begin{array}{l}\text { Genus des } \\
\text { Subst. }\end{array}$} & \multicolumn{5}{|c|}{$1650-1700$} & \multicolumn{5}{|c|}{$1700-1750$} & \multicolumn{5}{|c|}{$1750-1800$} \\
\hline & \multirow[t]{2}{*}{ zween } & \multirow[t]{2}{*}{ zwo } & \multicolumn{3}{|c|}{ zwei } & \multirow[t]{2}{*}{ zween } & \multirow[t]{2}{*}{$z w o$} & \multicolumn{3}{|c|}{ zwei } & \multirow[t]{2}{*}{ zween } & \multirow[t]{2}{*}{ zwo } & \multicolumn{3}{|c|}{ zwei } \\
\hline & & & $\mathbf{M}$ & $\mathbf{F}$ & $\mathbf{N}$ & & & $\mathbf{M}$ & $\mathbf{F}$ & $\mathbf{N}$ & & & $\mathbf{M}$ & $\mathbf{F}$ & $\mathbf{N}$ \\
\hline Norddt. & 7 & 2 & 5 & 2 & 8 & 8 & & 7 & 5 & 11 & 7 & 2 & 7 & 6 & 9 \\
\hline WMD & 9 & 6 & 10 & & 19 & 1 & 5 & 11 & 7 & 5 & 2 & 2 & 12 & 8 & 6 \\
\hline OMD & 4 & 2 & 12 & 3 & 3 & & & 4 & 5 & 5 & 2 & 2 & 19 & 1 & 5 \\
\hline WOD & 5 & 1 & 4 & 1 & 2 & 3 & 6 & 8 & 2 & 8 & 2 & 1 & 6 & 11 & 5 \\
\hline OOD & 13 & 2 & 6 & 3 & 10 & 11 & 1 & 10 & 7 & 7 & & 1 & 7 & 7 & 8 \\
\hline
\end{tabular}

Tab. 3: Genusdifferenzierung beim Zahlwort zovei

Jedoch empfahlen alle Grammatiker und Sprachtheoretiker des 18. Jahrhunderts bis Adelung die Genusdifferenzierung als korrektes Deutsch, vgl. Walch/Häckel (1988, S. 539). Gottsched (1762, S. 200-221) scheint sich z.B. wohl bewusst zu sein, dass er hier gegen den mehrheitlichen Gebrauch dafür plädiert, indem er zugibt, dass „das zweene, zwo, zwey“ manchem „fremd“" vorkommen möchte. Er unterstützt seine Präskription jedoch mit dem Argument, dies sei der Gebrauch der „Alten“ gewesen und in der Lutherschen Bibelübersetzung zu beobachten, d.h. er wendet als Kriterium ganz explizit den Mythos der althergebrachten Sprache an, und seiner Meinung nach obliegt es den heutigen Sprachteilhabern, den ererbten Formen- 
reichtum zu bewahren. Den Ausgleich der Unterschiede scheint Gottsched zumindest implizit als Verfall zu werten, aber seit frühneuhochdeutscher Zeit war sonst jegliche Genusdifferenzierung im Plural verschwunden beim bestimmten Artikel z.B. schon im 15. Jahrhundert, vgl. Ebert et al. (1993, S. 219), und die natürliche diachrone Entwicklung, wobei das Genus als morphosyntaktische Kategorie auf den Singular als den nicht markierten Numerus beschränkt war, war letztlich nicht durch das künstliche Einwirken der Sprachwächter aufzuhalten. In dieser Hinsicht sind daher die Belege für die Genusdifferenzierung in norddeutschen Texten im 18. Jahrhundert interessant, denn in den niederdeutschen Dialekten ist sie sehr früh verschwunden, vgl. Schirmunski (1962, S. 474). Vielleicht lässt sich dies auf eine genauere Beachtung der grammatischen Präskriptionen zurückführen in einer Region, wo das Hochdeutsche noch nicht zur Primärsprache geworden war. Aber dabei ist zu vermerken, dass alle Beispiele für die Genusdifferenzierung im späten 18. Jahrhundert einem einzigen Text entstammen, und zwar Herders Abhandlung über den Ursprung der Sprache vom Jahre 1772. Aber auch sein Gebrauch ist nicht konsequent, indem er auch genusindifferentes zwei verwendet.

\section{3 tun als Hilfsverb}

Es kommen jedoch auch Fälle vor, wo die Sprachwächter anscheinend doch den Gang der Entwicklung bestimmt haben, indem ihre Präskriptionen für die endgültig kodifizierte Hochsprache akzeptiert wurden. Wenn Sick (2006, S. 67) seine Meinung über die Inakzeptabilität des Gebrauchs von tun als Hilfsverb äußert, greift er auf eine lange Tradition zurück, wie Langer (2001) und Davies/Langer (2006, S. 211-224) gezeigt haben. Bei Sick (2006, S. 67) sehen wir wieder die Abwertung einer vermeintlichen „Simplifizierung“ der Grammatik, und die Charakterisierung dieser Konstruktion als „nicht elegant" oder „unbeholfen“ findet man schon früher. Diese Einschätzung ist natürlich rein subjektiv, denn man könnte sich kaum explizite linguistische Kriterien vorstellen, nach denen man eine regelmäßige grammatische Konstruktion so bezeichnen könnte. Aber der Gebrauch von tun als Hilfsverb wurde sehr früh mit ähnlicher Begründung für schlechtes Deutsch gehalten und als Beispiel für den Sprachverfall gewertet, wie z.B. von Zesen 1643 (Langer 2001, S. 189), dessen abwertende Bezeichnung von tun als „Flickwort" durchaus mit den von Sick für die Stigmatisierung dieser Konstruktion angeführten Gründen vergleichbar ist, aber Zesen appelliert darüber hinaus ausdrücklich an Mythen, denen wir schon begegnet sind, und zwar vor allem an den Mythos der reinen, althergebrachten Sprache, gegen deren Grundsätze dieser Gebrauch von tun verstoße, weil es ,nicht von Anbeginn in der Deutschen Sprache gewesen“" sei, vgl. Langer (2001, S. 189). In der Bezeichnung „Flickwort" erkennen wir auch den Versuch, die Präskrip- 
tion rationalistisch zu begründen, wie es für die zeitgenössische Auffassung von Sprache und Grammatik charakteristisch ist. Die Konstruktion mit tun ist überflüssig und steht daher im Widerspruch zu den Regeln der Vernunft, weil sie in der - allerdings irrtümlichen - Meinung dieser Sprachtheoretiker nichts anderes auszusagen vermag als das einfache Verb und keine selbstständige Funktion oder Bedeutung hat.

Langer (2001) zeigt, wie der Gebrauch von tun als Hilfsverb im Laufe des 17. und 18. Jahrhunderts mit derartigen Begründungen allmählich stigmatisiert wurde, und ab 1680 gilt es überhaupt als ,nicht korrekt", wobei ein weiterer Mythos nunmehr ins Spiel gebracht wird, und zwar die Vorstellung der „reinen“ Sprache des Nordens im Gegensatz zur „verderbten“ Sprache des Südens. Diese Ansicht finden wir z.B. in den Bemerkungen von Bödiker zum Gebrauch von tun als Hilfsverb, wo er die falsche Sprechweise der Schwaben und anderer Oberländer dem reinen und sauberen Sprachgebrauch des Nordens gegenüberstellt, vgl. Langer (ebd., S. 202). Nach 1740 wird dann nach Langer (2001) die Konstruktion zusätzlich soziolinguistisch stigmatisiert, als Merkmal der Pöbelsprache, aber diese Abwertung ist schon in den Äußerungen von Bödiker unverkennbar. Es ist auch durchaus typisch für den sprachlichen Standardisierungsprozess dieser Zeit, dass eine Variante, die aus irgendwelchem Grunde für die Hochsprache abgelehnt wird, als Merkmal der unteren, ungebildeten sozialen Schichten gekennzeichnet wird - ein Vorgang, den Reichmann (1988) als „Vertikalisierung“ des Variantenspektrums bezeichnet hat.

Diese Entwicklung der Stigmatisierung dieses Gebrauchs von tun kann aber nun mit dessen Vorkommen in unserem Korpus verglichen werden, das Langer (2001) natürlich noch nicht zur Verfügung stand. Die Konstruktion ist nicht häufig, mit insgesamt nur 59 Belegen, die zeitlich und regional sehr ungleichmäßig verteilt sind. In norddeutschen Texten kommt sie überhaupt nicht vor, und im Ostmitteldeutschen erscheint sie nur zweimal, in einem frühen Text, und zwar in Matthäus Hammers Rosetum Historiarum (Zwickau 1654). Sonst haben wir 7 Belege aus dem Westmitteldeutschen, 16 aus dem Ostoberdeutschen und 33 aus dem Westoberdeutschen, von denen nicht weniger als 22 einem einzigen Text entstammen, nämlich Johann Caspar Weissenbachs Aufnemmende Helvetia [...], einem in Zug aufgeführten volkstümlichen Schauspiel aus dem Jahre 1672. Sehr bezeichnend erscheint es jedoch auch, dass die Konstruktion nach 1710 lediglich dreimal im Korpus belegt ist, und von diesen stammt ein Beleg aus Philipp Hafners Wiener Komödie Mägera, die förchterliche Hexe aus dem Jahre 1764, und zwar bezeichnenderweise in einer Rede der Narrenfigur Hanswurst, in der der Autor deutlich einen volksnahen Ton intendiert hat:

HANSWURST: He! moderir sich der Herr, der Herr lacht wie ein Stockfisch, wenn der Herr wissen thäte, was in dem Namen Ganßbiegel stecket, so wurde der Herr anderst Respect haben. 
Aus diesem Material dürfen wir ein paar vorläufige Schlüsse ziehen, die nach weiterem Material zu ergänzen wären. Das Fehlen der Konstruktion mit tun in norddeutschen Texten lässt sich vielleicht dadurch erklären, dass sie von Schreibern mit niederdeutscher Muttersprache, die Hochdeutsch als Schreibsprache gelernt hatten, vermieden wurde. Sollte dies aber der Fall sein, so könnte man darauf hinweisen, dass die Konstruktion schon Mitte des 17. Jahrhunderts für weniger gut gehalten wurde - wir haben ja im Falle der Genusdifferenzierung von zwei gesehen, wie eine von den Grammatikern als mustergültig vorgeschriebene sprachliche Erscheinung in Norddeutschland im Schriftlichen aufgenommen wurde, obwohl sie im Niederdeutschen fehlte. Diese Annahme einer verhältnismäßig frühen Abneigung gegen die Umschreibung mit tun wäre dann durch die relativ spärliche Anzahl der Belege im Ostmitteldeutschen begründet, dessen schreibsprachlicher Gebrauch auch in anderen Regionen ein gewisses Prestige besaß. Darüber hinaus dürfte man aus der relativen Häufigkeit der Konstruktion in oberdeutschen Texten schließen, dass es sich sehr wohl um eine eher süddeutsche Schreibvariante handelt. So wäre die Stigmatisierung der umschriebenen Form nicht allein in abstrakten Überlegungen begründet, sondern man könnte sie in gewisser Hinsicht als den Versuch auffassen, einen im ostmitteldeutschen Raum schon etablierten Schreibusus mithilfe rationalistischer Argumente als ,korrekt“ zu rechtfertigen. Wie dem auch sei, in diesem Fall scheint die Bekämpfung eines vermeintlichen Sprachverfalls durch die Sprachwächter einigermaßen wirksam gewesen zu sein, indem die Konstruktion immer noch als nicht hochsprachlich gilt. Aber völlig verschwunden ist sie nicht. Anscheinend finden sie viele Muttersprachler in der gesprochenen Sprache immer noch nützlich, denn in bestimmten Kontexten greifen sie häufig zu dieser Umschreibung. Wie Langer (2001, S. 50-63) zeigt, gibt es wohl sprachliche Funktionen, die sich optimal dadurch ausführen lassen, was die Sprachwächter des 18. Jahrhunderts übersehen haben.

\subsection{Der Konditionalis mit würde}

Im Fall des sogenannten Konditionalis mit würde, den wir nun als nächstes (und letztes) Beispiel behandeln, sind die Grammatiker und Sprachtheoretiker nach Durrell (2007) anscheinend weniger erfolgreich gewesen. Der Konjunktiv ist ja stets einer ihrer Lieblingsgegenstände gewesen - nach Glück/Sauer (1997, S. 63) ist für sie der Konjunktiv ,,nicht nur ein Modus, er ist auch eine Weltanschauung“. Diese Ansicht lässt sich wohl wieder auf den Mythos der unveränderlichen althergebrachten Sprache zurückführen, denn anscheinend stellt man sich vor, dass alle Sprachen, die für sich eine ehrwürdige Geschichte in Anspruch nehmen wollen, wie Latein und Griechisch, über diesen schönen Modus verfügen müssen. So galt der Ersatz des syn- 
chronischen Konjunktiv Präteritum durch eine Umschreibung mit würde lange als klares Zeichen von Sprachverfall, und die Stigmatisierung dieser Konstruktion war im späten 19. und frühen 20. Jahrhundert besonders intensiv, vgl. Durrell (2007). Die Seiten der Zeitschrift für den deutschen Unterricht wimmeln von Klagen dagegen aus der Lehrerschaft, die charakteristische Elemente dieses Diskurses aufweisen, mit Hinweisen auf den Mythos der reinen unveränderlichen Sprache sowie mit typisch vagen und subjektiven Kriterien der vermeintlichen Sprachschönheit, wie z.B. in der folgenden Aussage von Maydorn (1892, S. 44):

Niemand wird es leugnen, daß es ein Zeichen des Verfalles ist, wenn alte klangvolle Formen zu vorzeitigem Absterben verdammt werden. [...] Ist man aber erst einmal gewöhnt, die alten schönen und kurzen Formen zu gebrauchen, dann bedarf es besonderer Belehrung nicht mehr, um die Unschönheit und Unzweckmäßigkeit der Umschreibungen einzusehen.

Auch fehlt in dieser Zeit des aufkommenden Nationalismus nach der Reichsgründung das patriotische Moment nicht, indem bei der Verbreitung dieser Konstruktion fremde Einflüsse gewittert werden, was natürlich mit dem Fremdwortpurismus dieser Zeit zusammenhängt. Scherffig (1905, S. 132) vermutet z.B. dass die Umschreibung mit würde ,unter dem Einfluß der französischen Sprache zu dem heutigen Umfang ihres Gebrauchs gekommen [ist]". Dass das Französische keine Konditionalparaphrasen mit einem Hilfsverb kennt, ist für dieses Argument offensichtlich nicht relevant. Auch der Mythos der „reinen“ Sprache des Nordens, der uns schon begegnet ist, darf in diesem Zusammenhang nicht fehlen, indem sich eine als nichthochsprachliche regionale Besonderheit des Südens angesehene Form aus ihrem ,angestammten heimischen Bezirk [erhob] zu einem Feldzug über den Main, eroberte und unterjochte den Norden und säete sich, während die Leute schliefen, als ein böses Unkraut unter unsern Weizen“ (Cüppers 1903, S. 297).

Diese Stigmatisierung findet sich dann in den Stilfibeln dieser Zeit, wie etwa Wustmann (1891, S. 184-185), wieder; aber entgegen deren Annahmen ist diese Paraphrase keineswegs neu und auch keine regionale Besonderheit. Sie lässt sich bis in frühneuhochdeutsche Zeit zurückverfolgen und ist in unserem Korpus in verschiedenen Verwendungsweisen reichlich belegt, als Ersatz für den synthetischen Konjunktiv Präteritum sowie auch in der Bedeutung eines Futurpräteritum. In dieser letzten Funktion, in der sie schon im ausgehenden Mittelalter belegt wurde, ist sie in unserem Material besonders häufig. Auch kommt sie im 17. und 18. Jahrhundert trotz der scharfen Stigmatisierung durch spätere Grammatiker nicht nur in der Protasis von Konditionalsätzen vor, sondern auch in der Apodosis, in diesem Fall mit einer erstaunlichen Häufigkeit in juristischen Texten. Auch in den Grammatiken des 18. Jahrhunderts findet man keinerlei Hinweise auf die Unrichtigkeit der Umschreibung mit würde, vgl. Auer (2009, S. 87-132). 
Adelung verwendet die Form reichlich in seinem eigenen Text und scheint ihren Gebrauch in allen Kontexten ohne Einschränkung zu akzeptieren. Insbesondere weist er darauf hin (Adelung 1782, \ 685), dass es keine Alternative dazu gibt, wenn man Zukünftiges in der Vergangenheit ausdrücken will. Erst in den 1830er Jahren, in den Grammatiken von Götzinger (1836) und Heyse (1838), finden wir eine klare Stigmatisierung, jedoch ohne jegliche Begründung außer der Behauptung, die Verwendung von würde verstoße ,gegen den guten hochdeutschen Sprachgebrauch“" und sei - wenn überhaupt - nur in der Apodosis zugelassen (ebd., S. 770-771). Die Belege in unserem Korpus weisen zwar darauf hin, dass die Paraphrase in der Protasis vor 1800 besonders häufig war und dass das synthetische Konjunktiv Präteritum etwas häufiger in der Apodosis verwendet wurde, aber diese Verteilung war keineswegs eine feste Regel, und es gibt überhaupt keine Indizien dafür, dass die Umschreibung vor den 1830er Jahren in irgendwelchen Kontexten systematisch vermieden, geschweige denn stigmatisiert wurde.

Wenn man von einer unerwünschten Neuerung sprechen will, dann kann man nur sagen, dass sie in der plötzlichen unbegründeten Stigmatisierung der wïrde-Form lag. Diese taucht in den 1830er Jahren wie aus dem Nichts auf, und die Präskription scheint dem eigentlichen Sprachgebrauch keineswegs entsprochen zu haben - weder in der geschriebenen noch in der gesprochenen Sprache - und sie entbehrte zunächst jeglicher Motivierung. Im Laufe des 19. Jahrhunderts wurde jedoch von den gebildeten Schichten akzeptiert, dass die Umschreibung mit würde nicht hochsprachlich sei, und diese Annahme wurde dann von den Sprachwächtern durch Begründungen rationalisiert, die aus den charakteristischen landläufigen Mythen über die Sprache abgeleitet wurden. Jedoch war diese Stigmatisierung letztlich gekünstelt, und sie hat sich infolge eines intensiven Drucks seitens der Lehrerschaft nur mit Mühe gegen den ererbten Sprachgebrauch behaupten können. Rückblickend mag es als lächerlich erscheinen, dass man die Umschreibung im späten 19. und frühen 20. Jahrhundert als beispielhaft für den Sprachverfall gehalten hat, aber die Einschätzung von Durrell (2007), dass die Stigmatisierung heutzutage endgültig am Ende sei, erscheint doch etwas verfrüht, denn es fehlt immer noch nicht an Versuchen, sie aufrechtzuerhalten. Ludwig Reiners' Stilfibel ist soeben 2013 in einer neuen Auflage erschienen, und dort wird der Gebrauch von wenn mit würde, also in der Apodosis, als „Stilgebrechen“ gewertet (Reiners 2013, S. 55-56). Dies sei „eine Regel [...], für die es keine logische Begründung“" gebe, aber es handele sich um eine Schulregel, an die wir uns halten müssten, obwohl es uns manchmal schwerfalle, sie zu befolgen. Nach Reiners beruhe diese Präskription „auf dem Sprachgebrauch“, aber wenn das so ist, versteht man kaum, warum es dann so schwierig sein sollte, die Regel zu befolgen. Dagegen nahm jedoch schon die erste bundesdeutsche Auflage der Duden-Grammatik aus dem Jahre 1959 (Duden 1959, S. 536) ganz klar dazu Stellung: 
Die Umschreibung muß heute überall als korrekt betrachtet werden, wo die einfache Konjunktivform veraltet ist, geschraubt klingt oder mit der Indikativform übereinstimmt. Sie ist nur dort überflüssig, wo die einfache Konjunktivform noch der natürlichen Sprechweise entspricht.

Eine durchaus vergleichbare Stellungnahme zu dieser Frage ist auch in allen späteren Auflagen der Grammatik zu finden, vgl. Duden (2009a, S. 538-539).

Aber auch im Dudenverlag kann man sich offensichtlich nicht vollständig von alten präskriptiven Gewohnheiten trennen: In dem DudenKalender „Auf gut Deutsch“ (Duden 2008) zum 27. Juli 2009 wird nämlich die Verwendung des synthetischen Konjunktiv Präteritum immer noch als „,korrekt“ empfohlen (allerdings mit dem Zugeständnis an fünfzehnjährige Mädchen, dass sie die Umschreibung vielleicht noch umgangssprachlich verwenden dürfen).

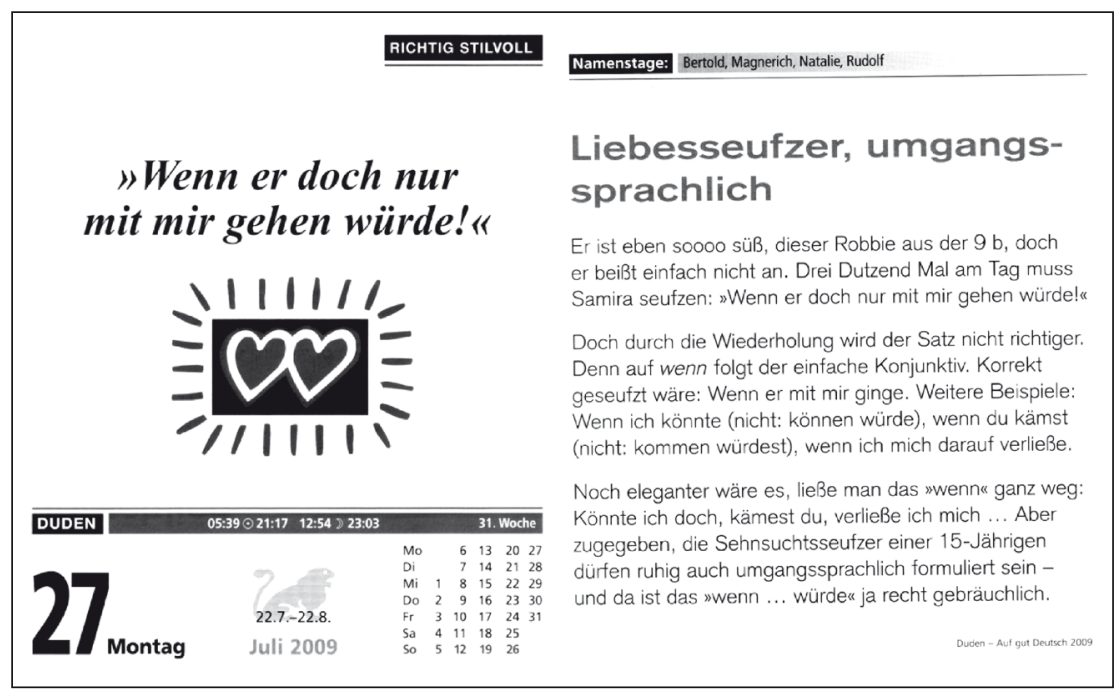

Abb. 1: Empfehlungen im Duden-Kalender 2009 zum Gebrauch von würde

Und in der nächsten Ausgabe dieses Kalenders (Duden 2009b) wurden zum 13./14. November weitere Beispiele gebracht, bei denen die Präskription, dass der Ersatz des Konjunktiv II durch Kombinationen mit würde möglichst vermieden werden soll, sehr klar zum Ausdruck kommt (Abb. 2).

Die Einschränkung ,nach Möglichkeit“" ist natürlich eine unaufrichtige Ausweichung, die über die Sprachwirklichkeit hinwegtäuscht, dass viele Formen des Konjunktiv II in der Tat veraltet sind und ,geschraubt klingen“. Im Kalender 2009 (Duden 2008) findet man am 2. Juli unter der Rubrik „Konjunktiv korrekt“" sogar folgende Empfehlung (Abb. 3): 
Manchmal scheint es, als habe das Deutsche die Umlaute hauptsächlich dazu erfunden, um seine Konjunktive bilden zu können. Doch, Hand aufs Herz, ist es nicht, als gewönne die Sprache dadurch erst an Farbe? Verlöre sie nicht Entscheidendes, bebülfe man sich nicht dieser Formen, nähme man sie nicht wahr und sprössen nicht alle möglichen ä-, ö- und ü-Schattierungen aus den Ritzen des Deutschen? Es läge uns also am Herzen, ergössen sich die Umlaute durchaus reichlich.

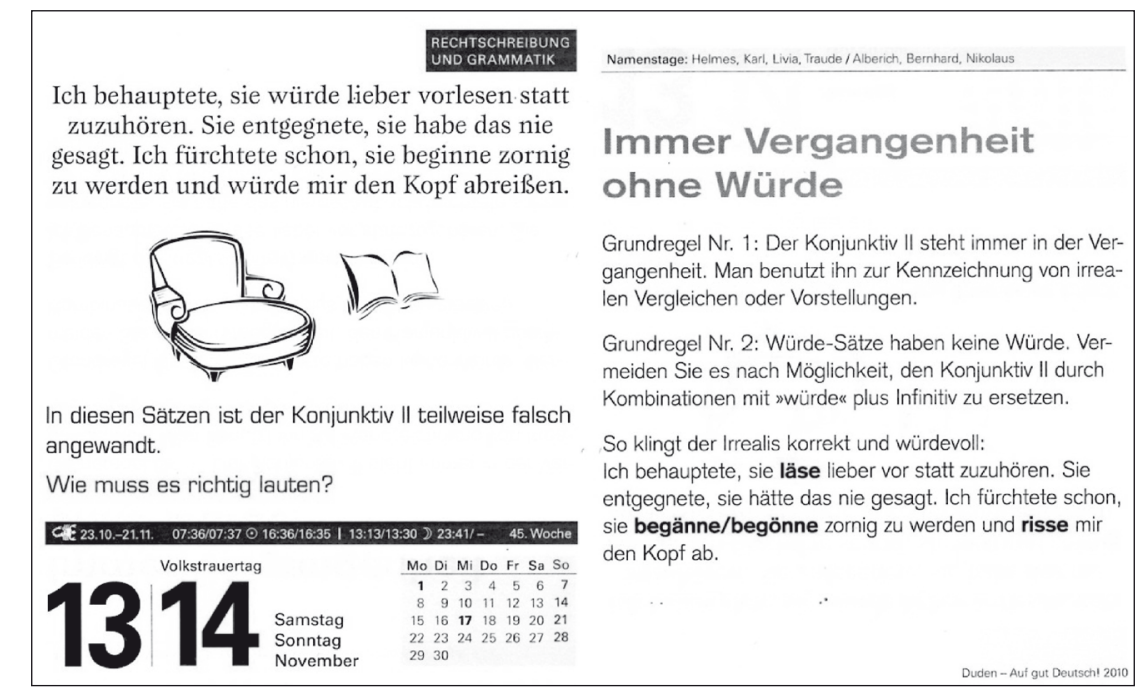

Abb. 2: Weitere Empfehlung zum Gebrauch von würde im Duden-Kalender 2010

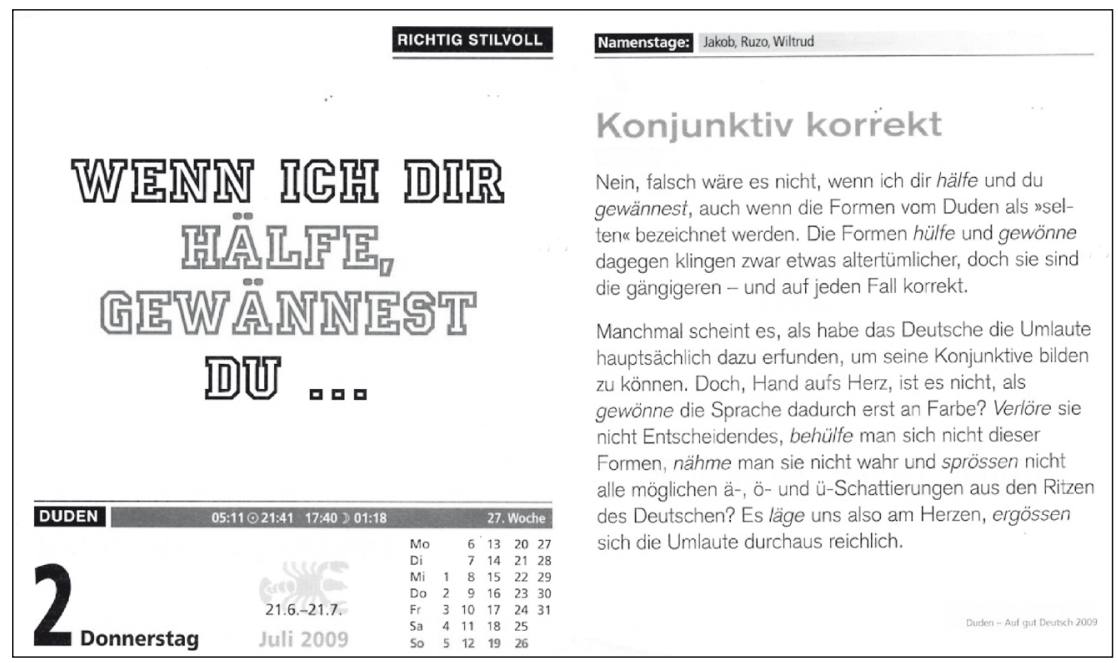

Abb. 3: Der Weisheit letzter Schluss? Weitere Empfehlungen im Duden-Kalender 2009 zum richtigen Gebrauch von würde 
Solche Formen können im heutigen Deutsch kaum als akzeptabel gelten, und dass sie vom Dudenverlag als „,korrekt" kolportiert werden sollten, kommt einer Persiflage gleich. Aber dass in einer Grammatik mit wissenschaftlichem Anspruch, wie Duden (2009a), etwas anderes behauptet wird als in laienlinguistischen Kalendern von demselben Verlag, veranschaulicht auf beispielhafte Weise die gespaltenen Einstellungen zum sprachlichen Wandel, die zur Verunsicherung bei den Sprachteilhabern und zu irrtümlichen Vorstellungen von einem Verfall der Sprache führen.

\section{Schlussfolgerungen und Fazit}

Durch diese Fallstudien sollte gezeigt werden, dass Annahmen über einen vermutlichen Sprachverfall auf einer Fehleinschätzung des dynamischen Prozesses des Sprachwandels beruhen, die aus der unreflektierten Akzeptanz einiger beharrlicher Mythen über das Wesen der Sprache entsteht, insbesondere des Mythos der grundsätzlichen Homogenität und Unveränderlichkeit der Sprache. Am Schluss ihrer Einführung in die historische Sprachwissenschaft mit dem bewusst provokativen Titel „Language Change: Progress or Decay?" gibt Aitchison (2013, S. 234) eine zusammenfassende Antwort auf die im Titel gestellte Frage, ob der sprachliche Wandel als Fortschritt oder Verfall zu werten sei, und sie kommt zu dem Schluss, dass weder das eine noch das andere zutrifft, denn:

The majority of self-proclaimed 'experts' who argue that language is disintegrating have not understood the complexity of the factors involved in language change. They are giving voice to a purely emotional expression of their hopes and fears.

Die hier aufgeführten Beispiele bestätigen durchweg diese Einschätzung. Die Sprache ist weder homogen noch unveränderlich, aber dem Sprachteilhaber fällt die ständige Variation im sprachlichen Alltag nicht als solche auf. Wie Maitz (2010, S. 2) neuerdings gesagt hat, sind Variation und Wandel „essentielle funktionelle Eigenschaften natürlicher menschlicher Sprachen“, sie sind nötig, damit die Sprache den Zweck der zwischenmenschlichen Kommunikation erfüllen kann. Man muss sich nämlich je nach Gesprächspartner, nach sozialer Situation oder nach Medium anders ausdrücken können. Die deutsche Sprache ist kein invariantes, für alle Zeiten festgelegtes, von den Sprechern unabhängiges Naturobjekt, sondern ein anpassungsfähiges Kommunikationsmittel, und muttersprachliche Sprecher sind völlig fähig, sie nach ihren empfundenen Bedürfnissen zu verwenden und weiter zu entwickeln. Wenn sie nicht mehr imstande wären, auf diese Weise die Sprache immer wieder nach den ihnen in der Struktur der Sprache zur Verfügung stehenden Mitteln neu zu gestalten, dann würde die Sprache nicht einfach in Verfall geraten, sie würde sterben. 
Diese Fallstudien aus der Sprachgeschichte zeigen jedoch, dass selbsternannte Sprachwächter - die ,guardians“ im Sinne von Joseph (1987) bzw. die oben erwähnten ,self-proclaimed 'experts" “ von Aitchison (2013, S. 234) - seit der frühen Neuzeit die von Watts $(2011,2012)$ aufgezeichneten Mythen als selbstverständlich hingenommen und natürliche sprachliche Entwicklungen zu beeinflussen oder zu bremsen versucht haben, auf der Basis von vagen subjektiven Kriterien, die sich ihrer Meinung nach daraus ableiten ließen bzw. die ihnen zu diesem Zwecke einfielen. Aber, wie Aitchison (2013, S. 234) sagt, sie sind mit diesen Bestrebungen selten absolut erfolgreich gewesen, denn natürliche Prozesse der sprachlichen Entwicklung lassen sich letztlich nicht aufhalten, und der Sprachgebrauch einer Mehrheit von Sprachteilhabern kann auf die Dauer nicht als inakzeptabel oder ,inkorrekt" stigmatisiert werden. Präskriptionen führen höchstens zur Verunsicherung oder aber auch zur Ausbildung der Variation, indem als „nicht korrekt" oder „nichthochsprachlich“ gebrandmarkte Alternativen in anderen weniger formellen sprachlichen Registern weiter verwendet werden und zu deren Charakterisierung beitragen.

Jedoch können solche Einsichten keineswegs als die Ergebnisse der neusten Forschung gelten. Sitta (1990) hat auf einer Jahrestagung des IDS vor über 20 Jahren vergleichbare Gedanken vorgetragen, und ähnliche Ansichten sind bei Polenz (2000, S. 19) zu finden. Aber fast ein Jahrhundert davor, im Jahre 1900, hat Otto Behaghel einen Vortrag im Freien Deutschen Hochstift in Frankfurt am Main gehalten über „Sprachgebrauch und Sprachrichtigkeit", in dem er sich mit genau derjenigen Problematik befasste, die das Hauptthema dieser Tagung bildet (Behaghel 1900). Er spricht mit sprudelndem Witz und tiefer Einsicht über die verbreitete, aber seiner Meinung nach total verkehrte Ansicht, dass die deutsche Sprache vor dem baldigen Verfall gerettet werden müsse, wobei er genau die Erscheinungen erwähnt, die noch heute bei Bastian Sick $(2004,2006)$ vorgeführt werden und die damals Gustav Wustmann (1891) beanstandete, wie z.B. größer wie oder größer als oder den Gebrauch des Dativs nach trot:. Er spricht von dem grundliegenden Unterschied zwischen dem mündlichen und dem schriftlichen Gebrauch sowie auch davon, dass vor allem in der gesprochenen Sprache von sprachlicher Einheitlichkeit keine Rede sein kann. Nach ihm müsse man erkennen, dass die sogenannten Sprachfehler und Schwankungen der Sprache etwas Berechtigtes, sogar etwas Notwendiges sind, und dass alle Sprachveränderungen darauf ausgehen, mit möglichster Kraftersparung möglichst vollkommen den Zweck der Sprache zu erreichen, nämlich die Verständigung zwischen den Menschen. Und zum vermeintlichen Verfall der Sprache sagte er Folgendes aus, wobei er sich auch auf die Kraft der Mythen bezieht, von denen hier die Rede war (Behaghel 1900, S. 19): 
Es gab eine Zeit, wo unsere germanistische Wissenschaft, voran ihr Begründer Grimm, des Glaubens war, daß im Leben der Sprache das Jüngere stets das Schlechtere sei; daß sprachliche Entwickelung ziemlich gleichbedeutend sei mit sprachlichem Verfall. [...] Und das ist auch der Standpunkt des Mannes, der vor kurzem sich zum Gesetzgeber der Sprache aufgeworfen hat, von Wustmann in seinen „Sprachdummheiten“. [...] Heute wissen wir, daß diese Anschauung vom Leben der Sprache eine durchaus verkehrte ist. Wie schon früher auf dem Gebiete der Naturwissenschaften, so hat man auch jetzt bei uns die Erkenntnis gewonnen, $\mathrm{da}$ in alten Zeiten genau die gleichen Ursachen gewirkt, die gleichen Vorgänge sich abgespielt und die gleichen Wirkungen sich ergeben haben, wie diejenigen, die wir in den lebenden Sprachen unmittelbar beobachten können. Es liegt im Wesen der Sprache, es ist eine unbedingte Naturnotwendigkeit, daß sie sich verändert, daß ihre Entwickelung in keinem Augenblick stille steht.

Am Beginn seines Vortrags bemerkt Behaghel (ebd., S. 16), dass diese Fragen oft mit einer Leidenschaftlichkeit behandelt werden, wie sie sonst nur bei politischen und wirtschaftlichen Fragen üblich ist, und dass die Heftigkeit des Streites sehr häufig im umgekehrten Verhältnis zu der Einsicht steht, mit der der Streit geführt wird.

Dazu kann man nur hinzufügen, dass wir in der Zwischenzeit anscheinend nicht so viel dazu gelernt haben, wie wir gern meinen möchten, weil man immer noch auf selbsternannte Sprachwächter und Experten wie damals Wustmann und jetzt Bastian Sick hört und, wie zu Jacob Grimms Zeiten, „des Glaubens zu sein scheint, dass sprachliche Entwickelung ziemlich gleichbedeutend sei mit sprachlichem Verfall“, und wir doch noch nicht richtig zur Kenntnis genommen haben, daß „diese Anschauung vom Leben der Sprache eine durchaus verkehrte ist" (Behaghel 1900, S. 19).

\section{Literatur}

Adelung, Johann Christoph (1782): Umständliches Lehrgebäude der deutschen Sprache, zur Erläuterung der deutschen Sprachlehre für Schulen. 2 Bde. Leipzig.

Aitchison, Jean (2013): Language change: Progress or decay? 4. Aufl. Cambridge.

Auer, Anita (2009): The Subjunctive in the Age of Prescriptivism. English and German developments during the Eighteenth Century. (= Palgrave Studies in Language History and Language Change). Basingstoke.

Behaghel, Otto (1900): Sprachgebrauch und Sprachrichtigkeit. In: Wissenschaftliche Zeitschrift des Allgemeinen Deutschen Sprachvereins 17/18, S. 16-30.

Bennett, Paul et al. (2011): Investigating diachronic grammatical variation in Early Modern German. Evidence from the GerManC corpus. In: Konopka, Marek et al. (Hg.): Grammatik und Korpora 2009. (= Korpuslinguistik und interdisziplinäre Perspektiven auf Sprache 1). Tübingen, S. 539-549. 
Cüppers, W. (1903): Mißbrauch der Umschreibung mit „würde“. In: Zeitschrift des Allgemeinen Deutschen Sprachvereins 18, S. 294-298.

Davies, Winifred V./Langer, Nils (2006): The making of bad language. Lay linguistic stigmatisations in German: Past and present. (= Variolingua 28). Frankfurt a.M. u.a.

Duden (1959): Der große Duden. Bd. 4: Grammatik der deutschen Gegenwartssprache. Hrsg. v. d. Dudenredaktion. Mannheim.

Duden (2001): Deutsches Universalwörterbuch. 4., neu bearb. u. erw. Aufl. Hrsg. v. Wissenschaftlichen Rat der Dudenredaktion. Mannheim u.a.

Duden (2008): Duden-Kalender „Auf gut Deutsch!““ 2009. Mannheim u.a.

Duden (2009a): Die Grammatik. Unentbehrlich für richtiges Deutsch. 8., überarb. Aufl. Hrsg. v. d. Dudenredaktion. Mannheim u.a.

Duden (2009b): Duden-Kalender „Auf gut Deutsch!“ 2010. Mannheim u.a.

Durrell, Martin (1999): Standardsprache in England und Deutschland. In: Zeitschrift für germanistische Linguistik 27, S. 285-308.

Durrell, Martin (2001): Nationalism and the history of the German national language. Theodor Frings's theories of the origin of standard German. In: Davies, Maire C./ Flood, John L./Yeandle, David N. (Hg.): ,Proper Words in Proper Places'. Studies in lexicology and lexicography in honour of William Jervis Jones. (= Stuttgarter Arbeiten zur Germanistik 400). Stuttgart, S. 195-212.

Durrell, Martin (2007): 'Deutsch ist eine würde-lose Sprache'. On the history of a failed prescription. In: Elspaß, Stefan et al. (Hg.): Germanic Language Histories 'from Below’. (= Studia Linguistica Germanica 86). Berlin/New York, S. 243-258.

Durrell, Martin/Ensslin, Astrid/Bennett, Paul (2008): Zur Standardisierung der Adjektivflexion im Deutschen im 18. Jahrhundert. In: Czachur, Waldemar/Czyżewska, Marta (Hg.): Vom Wort zum Text. Studien zur deutschen Sprache und Kultur. Festschrift für Professor Józef Wiktorowicz zum 65. Geburtstag. Warschau, S. 259-267.

Ebert, Robert P. et al. (1993): Frühneuhochdeutsche Grammatik. (= Sammlung kurzer Grammatiken germanischer Dialekte: A, Hauptreihe 12). Tübingen.

Fleischer, Jürg (2011): Historische Syntax des Deutschen. Eine Einführung. Tübingen.

Glück, Helmut/Sauer, Werner Wolfgang (1997): Gegenwartsdeutsch. 2., überarb. u. erw. Aufl. (= Sammlung Metzler 252). Stuttgart.

Gottsched, Johann Christoph (1762): Vollständige und Neuerläutete Deutsche Sprachkunst, Nach den Mustern der besten Schriftsteller [...] abgefasset. 5. Aufl. Leipzig.

Götzinger, Max Wilhelm (1836): Die deutsche Sprache und ihre Literatur. Bd. 1: Die deutsche Sprache. Erster Theil. Stuttgart.

Gutjahr, Emil A. (1910): Die Anfänge der neuhochdeutschen Schriftsprache vor Luther. Streifzüge durch die deutsche Siedelungs-, Rechts- und Sprachgeschichte auf Grund der Urkunden deutscher Sprache. Halle a.d.S.

Haugen, Einar (1966): Language, dialect, nation. In: American Anthropologist 68, S. 922-935. 
Heyse, Johann Christian August (1838): Theoretisch-praktische deutsche Grammatik oder Lehrbuch der deutschen Sprache nebst einer Geschichte derselben. Zunächst zum Gebrauch für Lehrer und zum Selbstunterricht. 5. völlig umgearb. u. sehr verm. Ausgabe von Karl Wilhelm Ludwig Heyse. Hannover.

Joseph, John Earl (1987): Eloquence and power. The rise of language standards and standard languages. (= Open Linguistics Series). London.

Keller, Rudolf Ernst (1978): The German language. London.

Kluge, Friedrich (1904): Von Luther bis Lessing. Sprachgeschichtliche Aufsätze. 4., durchges. Aufl. Straßburg.

Langer, Nils (2001): Linguistic purism in action. How auxiliary tun was stigmatized in Early New High German. (= Studia Linguistica Germanica 60). Berlin/New York.

Maitz, Péter (2010): Sprachpflege als Mythenwerkstatt und Diskriminierungspraktik. In: Aptum. Zeitschrift für Sprachkritik und Sprachkultur 6, S. 1-19.

Maydorn, Bernhard (1892): Über die Konjunktiv-Umschreibung mit „würde“. In: Zeitschrift für den deutschen Unterricht 6, S. 44-48.

Milroy, James/Milroy, Lesley (1999): Authority in language. Investigating language prescription and standardisation. 3. Aufl. London.

Polenz, Peter von (2000): Deutsche Sprachgeschichte vom Spätmittelalter bis zur Gegenwart. Bd. 1: Einführung, Grundbegriffe, 14. bis 16. Jahrhundert. 2., überarb. u. erg. Aufl. Berlin/New York.

Reichmann, Oskar (unter Mitwirkung von Christiane Burgl, Martin Kaufhold und Claudia Schäfer) (1988): Zur Vertikalisierung des Varietätenspektrums in der jüngeren Sprachgeschichte des Deutschen. In: Munske, Horst Haider et al. (Hg.): Deutscher Wortschatz. Lexikologische Studien. Ludwig Erich Schmitt zum 80. Geburtstag von seinen Marburger Schülern. Berlin/New York, S. 151-180.

Reiners, Ludwig (2013): Stilfibel. Der sichere Weg zum guten Deutsch. 4. Aufl. München.

Scherffig, Richard (1905): Zur Umschreibung mit „würde““. In: Zeitschrift für den deutschen Unterricht 19, S. 131-133.

Schirmunski, Viktor M. (1962): Deutsche Mundartkunde. Vergleichende Laut- und Formenlehre der deutschen Mundarten. (= Veröffentlichungen des Instituts für deutsche Sprache und Literatur 25). Berlin.

Sick, Bastian (2004): Der Dativ ist dem Genitiv sein Tod. Ein Wegweiser durch den Irrgarten der deutschen Sprache. Köln.

Sick, Bastian (2006): Der Dativ ist dem Genitiv sein Tod. Folge 3: Noch mehr Neues aus dem Irrgarten der deutschen Sprache. Köln.

Sitta, Horst (1990): Defizit oder Entwicklung. Zum Sprachstand von Gymnasialabsolventen und Studenten. In: Stickel, Gerhard (Hg.): Deutsche Gegenwartssprache. Tendenzen und Perspektiven. (= Jahrbuch des Instituts für deutsche Sprache 1989). Berlin/New York, S. 233-254. 
Solms, Hans-Joachim/Wegera, Klaus-Peter (1991): Grammatik des Frühneuhochdeutschen. Bd. 6: Flexion der Adjektive. (= Germanische Bibliothek: Reihe 1, Sprachwissenschaftliche Lehr- und Elementarbücher). Heidelberg.

Voeste, Anja (1999a): Varianz und Vertikalisierung. Zur Normierung der Adjektivdeklination in der ersten Hälfte des 18. Jahrhunderts. (= Amsterdamer Publikationen zur Sprache und Literatur 140). Amsterdam/Atlanta.

Voeste, Anja (1999b): Ist bei der Normierung der Adjektivdeklination in der ersten Hälfte des 18. Jahrhunderts von einer Vorbildwirkung ostmitteldeutscher Texte auszugehen? In: Bister-Broosen, Helga (Hg.): Beiträge zur historischen Stadtsprachenforschung. (= Schriften zur diachronen Sprachwissenschaft 8). Wien, S. 67-78.

Walch, Maria/Häckel, Susanne (1988): Grammatik des Frühneuhochdeutschen. Beiträge zur Laut- und Formenlehre. Bd. 7: Flexion der Pronomina und Numeralia. (= Germanische Bibliothek: Reihe 1, Sprachwissenschaftliche Lehr- und Elementarbücher). Heidelberg.

Watts, Richard J. (2011): Language myths and the history of English. (= Oxford Studies in Sociolinguistics). Oxford.

Watts, Richard J. (2012): Language myths. In: Hernández Campoy, Juan/Conde Silvestre, Juan Camilo (Hg.): The handbook of historical sociolinguistics. Malden, MA, S. 585-606.

Wustmann, Gustav (1891): Allerhand Sprachdummheiten. Kleine deutsche Grammatik des Zweifelhaften, des Falschen und des Häßlichen. Ein Hilfsbuch für alle die sich öffentlich der deutschen Sprache bedienen. Leipzig.

Zifonun, Gisela/Hoffmann, Ludger/Strecker, Bruno (1997): Grammatik der deutschen Sprache. (= Schriften des Instituts für Deutsche Sprache 7). Berlin/New York. 
